\title{
The lupus band test in systemic lupus erythematosus patients
}

This article was published in the following Dove Press journal:

Therapeutics and Clinical Risk Management

21 January 20II

Number of times this article has been viewed

\section{Adam Reich \\ Katarzyna Marcinow \\ Rafal Bialynicki-Birula \\ Department of Dermatology, Venereology, and Allergology, Wroclaw Medical University, Wroclaw, Poland}

Correspondence: Adam Reich Department of Dermatology, Venereology, and Allergology, Wroclaw Medical University, UI. Chalubinskiego I, 50-368 Wroclaw, Poland

Tel +48 605076722

Fax +48 7I 3270942

Email adi_medicalis@go2.pl

\begin{abstract}
The lupus band test (LBT) is a diagnostic procedure that is used to detect deposits of immunoglobulins and complement components along the dermoepidermal junction in patients with lupus erythematosus (LE). The LBT is positive in about $70 \%-80 \%$ of sun-exposed nonlesional skin specimens obtained from patients with systemic LE (SLE), and in about 55\% of SLE cases if sun-protected nonlesional skin is analyzed. In patients with cutaneous LE only, the lesional skin usually shows a positive LBT. The LBT helps in differentiating LE from other similar skin conditions and may also be helpful in making the diagnosis of SLE in subjects with no specific cutaneous lesions. Furthermore, a positive LBT may be applied as a prognostic parameter for LE patients. However, the correct interpretation of this test requires detailed knowledge of the site of the biopsy, deposit components, morphology and brightness of the immunofluorescent band, and other associated serologic findings, as well as the response to treatment. It must be emphasized that LBT is a laboratory procedure that should always be interpreted in conjunction with clinical findings and other serological and immunopathological parameters.
\end{abstract}

Keywords: lupus erythematosus, dermoepidermal junction, diagnostics

\section{Introduction}

Lupus erythematosus (LE) is an autoimmune disease affecting different body systems, including, but not limited to, joints, skin, kidneys, blood, heart, and brain, and may be characterized by the production of various autoantibodies, complement consumption, and the presence of circulating immune complexes. ${ }^{1}$ As in other autoimmune diseases, the autoimmune reaction in LE, driven by various components of the immune system, results in inflammation and tissue damage. Systemic LE (SLE), cutaneous LE (CLE) (including subacute CLE [SCLE] and discoid LE [DLE]), drug-induced LE, and neonatal LE are the major subtypes of LE. Of these, SLE is the most serious form. It occurs about 10 times more often among women than among men, usually in young adults, and Black and Asian people seem to be more commonly affected. ${ }^{2,3}$ This is a life-threatening disease, sometimes with a fatal outcome. Fortunately, the medical improvement of patients' care in the past few decades makes treatment failure increasingly rare. Currently, the 10-year survival rate in developed countries is estimated to be above $90 \%{ }^{4,5}$

The histology of the diseased skin of LE patients shows some typical characteristics, including vacuolar degeneration of the basal layer with thickened subepidermal basement membrane, as well as perivascular and periadnexal lymphocytic infiltrates, which may help to establish the diagnosis. ${ }^{1,6}$ In addition, lesional and nonlesional skin may 
show deposits of immunoglobulins along the dermoepidermal junction (DEJ), which is a unique feature of LE. ${ }^{6}$

\section{Definition of lupus band test (LBT)}

Deposits of immunoglobulins at the DEJ, together with the thickened basement membrane, in lesional skin of LE patients were first described by Burnham et al. ${ }^{7}$ Subsequently, Cormane $^{8}$ demonstrated similar deposits in clinically normal skin of SLE, but not in that of CLE subjects. Detection of these deposits of immunoglobulins and complement components in the skin of patients with LE, demonstrable as a linear band at the basement membrane zone, was then named the lupus band test (LBT). All major immunoglobulin classes (IgG, IgM, and IgA) and various complement components have been identified in these DEJ deposits. ${ }^{6}$ The test is done on the skin biopsy, usually with direct immunofluorescence staining, but immunohistochemistry may also be applied. Importantly, LBT can be helpful in distinguishing SLE from CLE, because in SLE patients the LBT is frequently positive in both involved and uninvolved skin, whereas in CLE patients only the involved skin is positive.

Although the exact mechanism of the immunoglobulin deposition at DEJ in LE patients is not clear, it is believed that these immunoglobulins are not antibodies against basement membrane zone components but rather represent, at least partly, circulating immune complexes of DNA and antinuclear antibodies trapped within the DEJ. Furthermore, DNA released from ultraviolet-injured keratinocytes, although diffusing across the basement membrane zone, may bind to collagen IV and then serve as an antigen for circulating antinuclear antibodies. ${ }^{6,9}$

\section{Sensitivity and specificity of the LBT}

The sensitivity and specificity of the LBT are strictly related to the body area tested and the criteria used for assigning the test as positive. For instance, in the study by Cardinali et al ${ }^{10}$ on the sun-protected nonlesional skin of SLE patients, the sensitivity of the LBT varied between $10.5 \%$ and $78.9 \%$ and the specificity between $47.8 \%$ and $97.8 \%$, depending on the criteria used by the authors. The most frequent immunoglobulin class deposited is IgM, which is seen in about $90 \%$ of lesional skin biopsies, whereas the least frequently seen class is IgA. ${ }^{6,11}$ However, a weak decoration with IgM along the DEJ is also common in sun-exposed skin of patients who do not have LE. It was demonstrated that healthy sun-exposed skin may show a weak interrupted linear or granular pattern of IgM and C1q deposits at the DEJ in nearly $25 \%$ of samples, but only $5 \%$ of them showed the presence of $\operatorname{IgG}, \operatorname{IgA}$, or complement component 3 (C3). ${ }^{12,13}$ Taking these observations into account, in line with Crowson and Magro, ${ }^{6}$ we suggest that LBT should be considered positive when deposits of IgM in sun-exposed skin form a continuous band over at least $50 \%$ of the width of the biopsy specimen which is at least moderate in intensity. The predictive value of LBT for SLE was greater with C4 (100\%), properdin (91.3\%), and IgA (86.2\%) than with $\operatorname{IgM}(59 \%) .{ }^{6}$ The specificity and predictive value also increase with the number of immunoreactants detected at the DEJ. ${ }^{6}$ In sun-protected skin, an interrupted band of IgM of at least moderate intensity is usually sufficient for designation as a positive LBT. ${ }^{6}$ The deposition of $\mathrm{IgG}$ is usually less intense than that of $\operatorname{IgM}$, and false-positive results are practically nonexistent. ${ }^{6}$

Altogether, the LBT in patients with SLE is positive in about $70 \%-80 \%$ subjects when sun-exposed nonlesional skin is tested, and in about $55 \%$ cases when sun-protected nonlesional skin is studied. In patients with CLE, the LBT of nonlesional skin is usually negative, although the lesional skin may frequently show immune deposits at the DEJ. ${ }^{6}$ Moreover, a positive LBT may serve as a prognostic indicator in patients with an established diagnosis of LE, as it correlates with severe extracutaneous disease, mainly lupus nephritis, and with anti-dsDNA antibodies. ${ }^{6,11,14,15}$

\section{Staining patterns of the LBT}

The immunoglobulin staining pattern in nonlesional LE skin at low magnification is usually described as granular or closely spaced vertically oriented fibrils, and sometimes also as a thick, homogeneous band (Figure 1). The C3 deposits are the most common component, and IgM was the most frequent immunoglobulin class observed in the LBT. ${ }^{16}$ Remarkably, a sharply defined, thin linear band at the DEJ seen in the pemphigoid should not be considered as a positive LBT (Figure 2). ${ }^{6}$ Under high power, the patterns of immunoglobulin deposition at the DEJ may be homogenous (Figure 3), fibrillar, stippled (Figure 4), shaggy (Figure 5), lumpy (Figure 6), linear, or thready. ${ }^{6,11}$ All these patterns are seen in a continuous fashion. A discontinuous or interrupted LBT is less specific and can be seen in a number of other disorders such as actinic keratosis, polymorphic light eruption, and rosacea and in normal sun-exposed skin (Table 1). ${ }^{11}$ Ultrastructurally, these immunoreactants are deposited on or below lamina densa of the basement membrane. ${ }^{11}$

A stippled pattern of the LBT, consisting of multiple small round points of fluorescence (Figure 4), is the type most commonly seen in clinically normal skin of SLE. In some 
Table I Differential diagnosis of lupus band test in lupus erythematosus from other conditions

\begin{tabular}{ll}
\hline Clinical condition & Comments \\
\hline Positive lupus band test & $\begin{array}{l}\text { Granular band or closely spaced, } \\
\text { vertically oriented fibrils } \\
\text { at dermoepidermal junction; sometimes } \\
\text { a thick, homogeneous band. } \\
\text { May demonstrate positive lupus } \\
\text { band test. } \\
\text { The band is usually less intensive } \\
\text { and frequently focal or interrupted. } \\
\text { No reactivity is seen in sun-protected } \\
\text { skin. } \\
\text { Might simulate a positive lesional lupus } \\
\text { band test. } \\
\text { Autofluorescence } \\
\text { of dermal collagen } \\
\text { and elastin fibers } \\
\text { finding becomes apparent at higher } \\
\text { magnification. } \\
\text { Sharply defined thin linear band } \\
\text { at dermoepidermal junction. } \\
\text { Presence of circulating antibodies against } \\
\text { basement membrane components } \\
\text { is helpful for a correct diagnosis. } \\
\text { Fluorescence of the dermoepidermal } \\
\text { junction is less intense than that found } \\
\text { in dermal blood vessels, which is exactly } \\
\text { the reverse of what is seen in lupus } \\
\text { erythematosus. } \\
\text { Complement is rarely found in the band. } \\
\text { The band is less intensive and frequently } \\
\text { focal or interrupted. } \\
\text { The band is less intensive and frequently } \\
\text { focal or interrupted. }\end{array}$ \\
\end{tabular}

patients, sparser, more elongated and threadlike stipples can be seen. A homogenous or solid band of well demarcated, bright fluorescence is seen mostly in chronic atrophic or hypertrophic skin lesions. A thready pattern consisting of

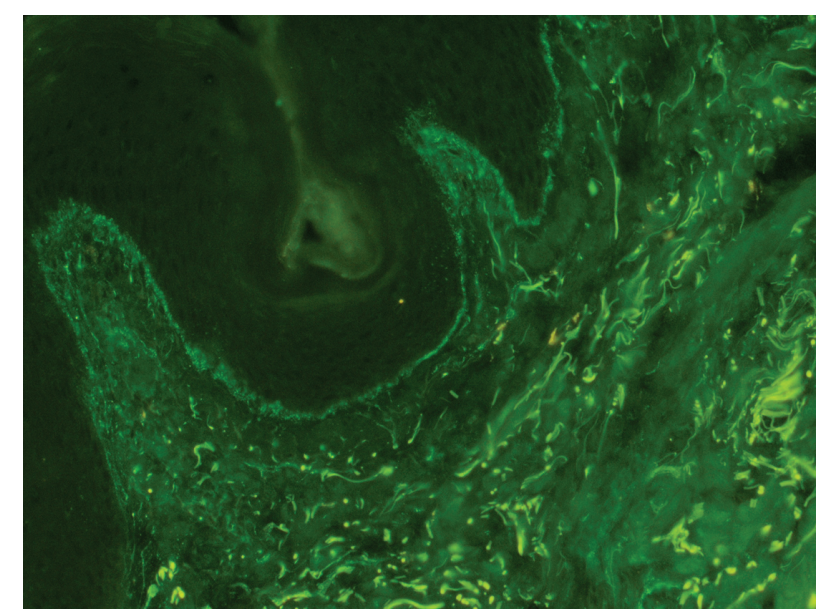

Figure I Positive lupus band test at low magnification: immunoglobulin class $M$ deposits at the dermoepidermal junction in sun-protected nonlesional skin in a 26-year-old woman with systemic lupus erythematosus (original magnification: $\times 100$ ).

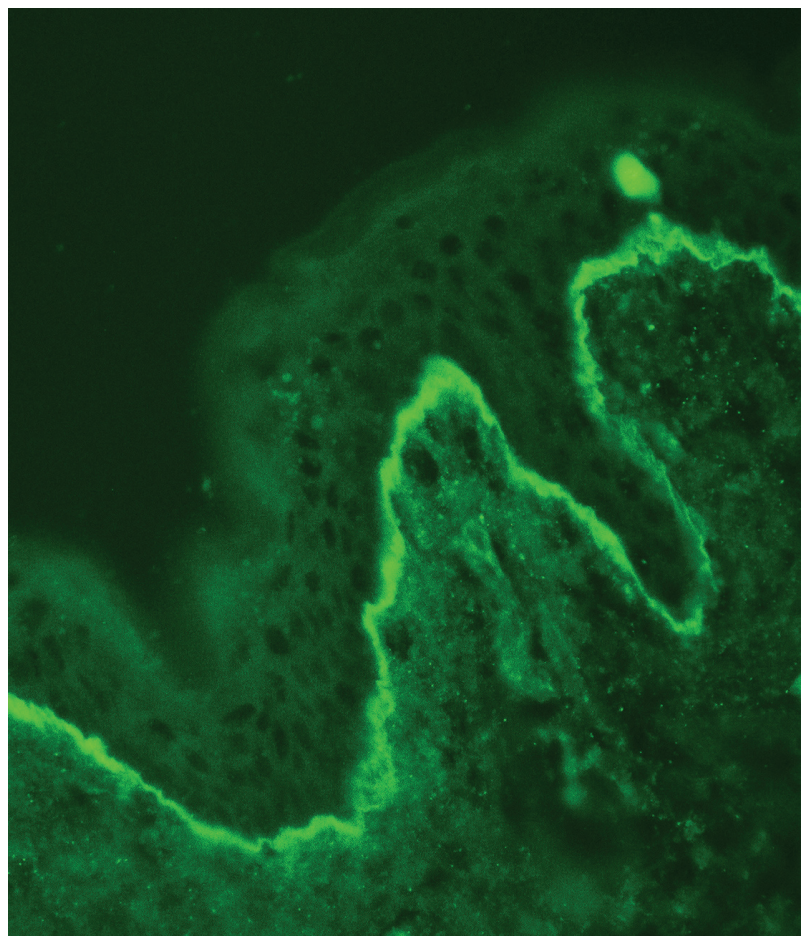

Figure 2 A sharply defined thin linear band at the dermoepidermal junction in pemphigoid (immunoglobulin class $G$ deposits, original magnification: $\times 200$ ).

short, closely set, bright threads or fibrils is seen in more acute erythematous, edematous lesions. Shaggy, fibrillar, lumpy, and granular patterns have also been seen, usually in a continuous pattern along the DEJ. ${ }^{11}$

Sometimes, a false-negative reaction can be observed due to high levels of extravascular IgG deposits in the dermis. ${ }^{11}$ Moreover, considerable anatomic regional variations may occur in positive lesional LBT with a cephalocaudal gradient,

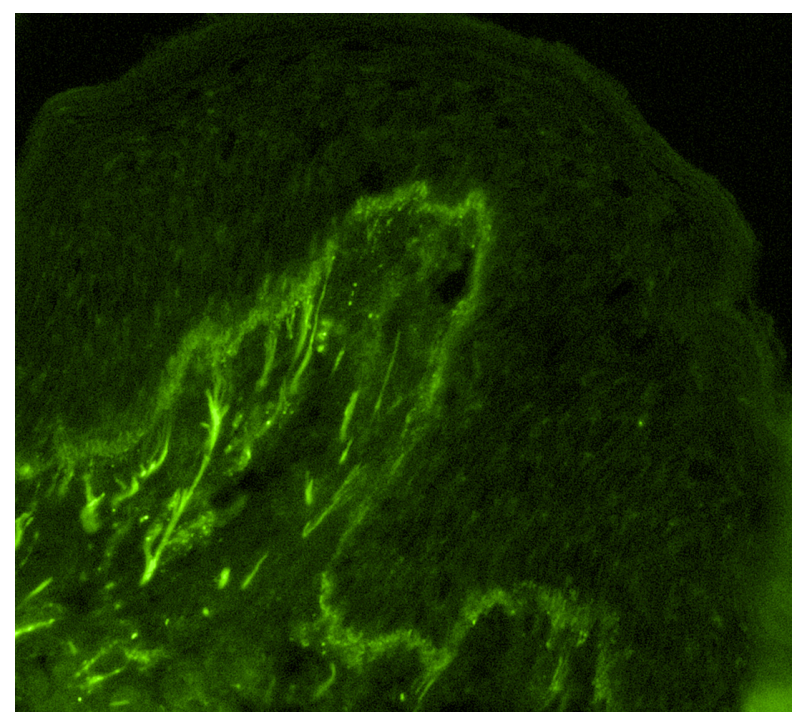

Figure 3 Partially homogenous, partially granular pattern of the lupus band test (immunoglobulin class $M$ deposits, original magnification $\times 200$ ). 


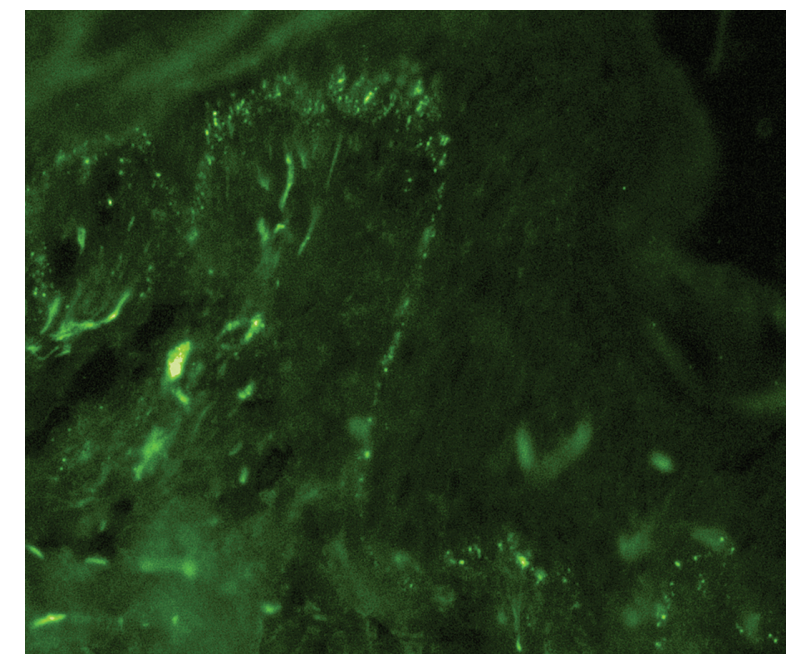

Figure 4 Stippled pattern of complement component 3 deposits in sun-protected nonlesional skin in patient with systemic lupus erythematosus (original magnification: $\times 400)$.

with lesions on the head more often being positive than those on the trunk. ${ }^{11}$

\section{Other staining methods in LE}

Detection of the $\mathrm{C}_{5 \mathrm{~b}-9}$ macromolecular structure, which is formed following activation of the classical or alternative component pathway, is another useful tool for the diagnosis of LE. ${ }^{1}$ Intense granular deposition of $\mathrm{C}_{5 \mathrm{~b}-9}$ along the $\mathrm{DEJ}$ is seen in about $80 \%$ of lesional skin in patients with SLE. ${ }^{17}$ Vascular decoration for $\mathrm{C}_{5 \mathrm{~b}-9}$ was observed in SLE subjects with anti-Ro/SSA antibodies, in patients with circulating

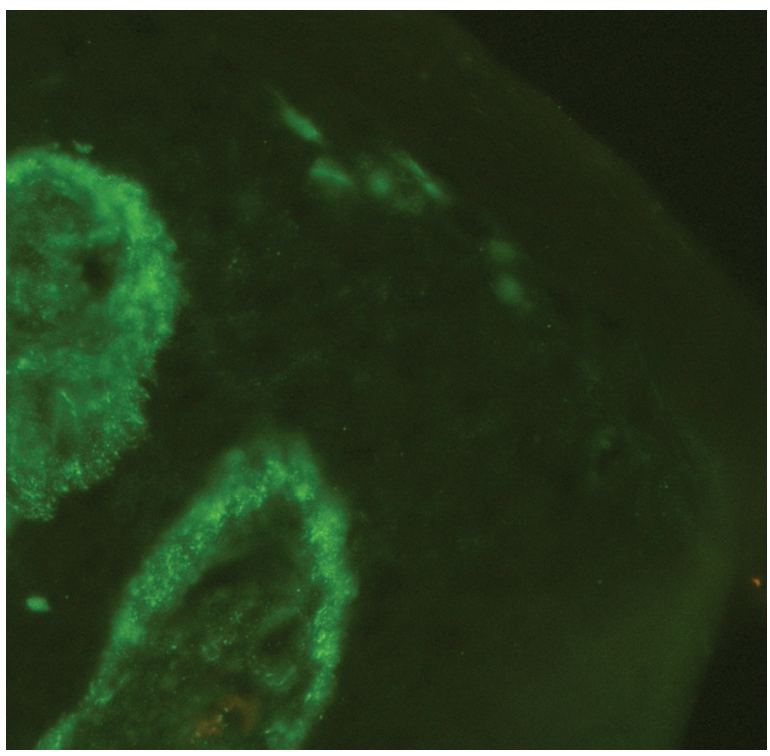

Figure 5 Shaggy pattern of complement component 3 deposits in sun-protected lesional skin in patients with subacute cutaneous lupus erythematosus.

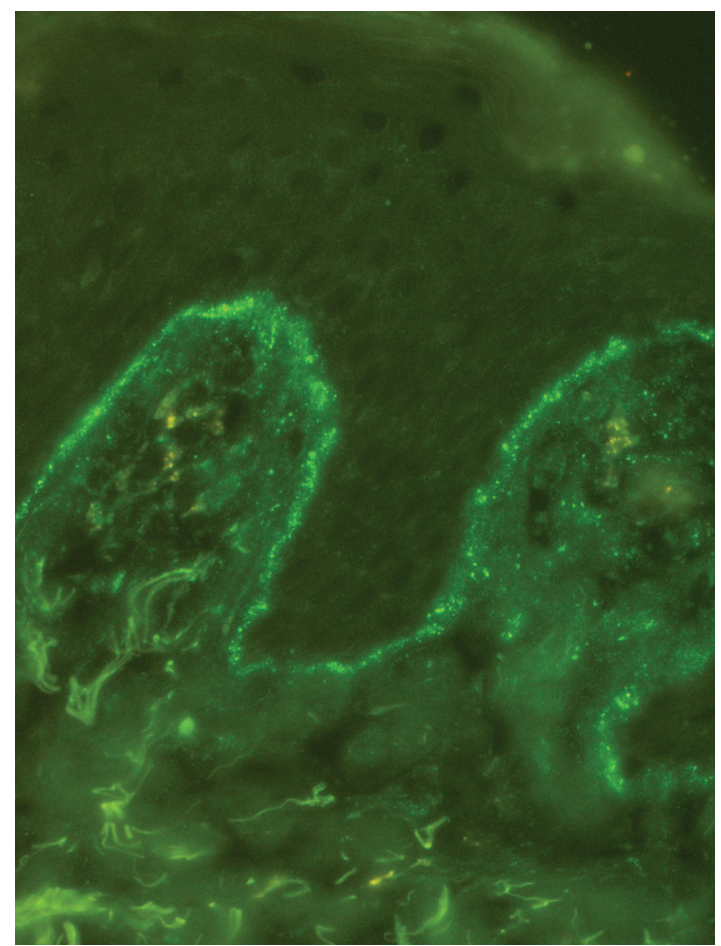

Figure 6 Granular (lumpy) pattern of complement component 3 deposits in sunprotected nonlesional skin in patient with systemic lupus erythematosus (original magnification: $\times 400$ ).

lupus anticoagulant, or when vasculitis is demonstrated in the biopsy. ${ }^{1}$ Regarding nonlesional skin of SLE patients, $\mathrm{C}_{5 \mathrm{~b}-9}$ immunoreactivity might be observed within keratinocytes, especially in subjects with positive anti-ENA antibodies (ENA: Ro, La, Sm, U1RNP). In SCLE and DLE, deposition of $\mathrm{C}_{5 \mathrm{~b}-9}$ along the DEJ was observed in $66 \%$ and $60 \%$ of cases, respectively. ${ }^{18}$ However, in SCLE, keratinocytes may show granular nuclear or cytoplasmic reactivity for $\mathrm{C}_{5 \mathrm{~b}-9}$, a finding that is usually absent in DLE. ${ }^{1,18}$

Recently, it was also shown that detection of $\mathrm{C} 3 \mathrm{~d}$ and $\mathrm{C} 4 \mathrm{~d}$ by immunohistochemistry might be used for diagnosis of LE as well. ${ }^{18} \mathrm{C} 4 \mathrm{~d}$ is a stable component of classical complement activation and represents a degradation product of C4b. ${ }^{19}$ $\mathrm{C} 3 \mathrm{~d}$ is also a stable component of complement activation, although it can be formed in both classical or alternative complement activation. ${ }^{19}$ As shown by Magro and Dyrsen, ${ }^{19}$ all DLE cases showed granular, moderate to marked intensity C3d deposits at DEJ, whereas C4d deposits were found in only $15 \%$ of DLE patients. Interestingly, all SCLE subjects were negative for $\mathrm{C} 3 \mathrm{~d}$ and $\mathrm{C} 4 \mathrm{~d}$ staining along the $\mathrm{DEJ}$, although some of them showed deposition of C3d or C4d in blood vessels, a finding that was also noted in DLE. Similarly to DLE, all samples of SLE demonstrated prominent granular C3d deposition along the DEJ. Remarkably, all SLE patients also had C4d deposits at the DEJ. ${ }^{19}$ 


\section{Application of the LBT}

The usefulness of the LBT as a diagnostic procedure in LE patients is well established. The positive result of this test within the uninvolved skin is a strong indicator of LE. ${ }^{11}$ The sensitivity of the LBT for diagnosing an active disease seems to be even higher than the value of other laboratory parameters, including serum $\mathrm{C} 3$ and $\mathrm{C} 4$ levels, erythrocyte sedimentation rate, lymphocyte count, or the presence of anti-dsDNA antibodies (the last two parameters are included in the diagnostic criteria of SLE).

The diagnostic specificity of LBT for LE differentiation from other inflammatory cutaneous conditions that are clinically similar, such as polymorphic light eruption or benign lymphocytic infiltrate of Jessner and Kanoff, is also high. ${ }^{11,16}$ Furthermore, a positive LBT in fully sun-protected nonlesional skin from the buttocks or inner aspect of the upper arm consisting of three or more components (IgA, IgG, IgM, or complement proteins) seems to have the highest specificity for SLE than any other test. ${ }^{11}$

The LBT may also be helpful in distinguishing SLE from CLE. Various studies have shown that SLE subjects have immunoglobulin and/or complement deposits at the DEJ in both involved and uninvolved skin, whereas CLE patients had only positive LBT in lesional skin. Therefore, it is essential to biopsy the appropriate skin site for each patient in order to avoid false-negative results. Furthermore, the LBT can be helpful in making a diagnosis of SLE in patients without cutaneous lesions. A positive LBT in clinically normal skin provides early confirmation of SLE, even in patients without LE lesions, where neither a biopsy nor LBT can be performed on a skin lesion. ${ }^{11}$ Importantly, test positivity was uninfluenced by steroid and immunosuppressive therapy. ${ }^{20}$ The test appears to be of great interest for use on all patients who fail to meet the criteria for the diagnosis of SLE but whose conditions suggest such diagnosis. ${ }^{20}$ In addition, the LBT facilitates differentiation of diagnosis of SLE from other antinuclear antibody-positive diseases like rheumatoid arthritis, scleroderma, dermatomyositis, and mixed connective tissue disease. ${ }^{1,11}$ However, occasional positive LBTs were found in lesional skin of dermatomyositis and systemic sclerosis, and this finding has been correlated with a more severe disease course. ${ }^{18,21}$

A positive LBT may also be a predictive value for the prognosis of LE patients. The deposition of IgG in nonlesional sun-protected skin is correlated with anti-dsDNA antibodies titer and with higher incidence of renal disease, although a negative LBT does not necessarily exclude the possibility of renal involvement. ${ }^{11,20,22}$ A positive LBT within sun-protected normal skin also indicates decreased long-term survival. ${ }^{11,22}$ Recently, Zecević et al, using the SLE Disease Activity Index score, confirmed that the disease was significantly more active in patients with positive LBT on sun-protected nonlesional skin and in those with a higher number of deposited immunoreactants. Moreover, almost all patients with renal involvement had a positive LBT. $^{23}$ On the other hand, patients with pure IgM deposition in clinically normal skin have anti-dsDNA antibodies restricted to the IgM class and tend to have a less severe disease course. In addition, $\mathrm{C} 1 \mathrm{q}$ deposits along the DEJ in the skin of SLE patients may reflect the presence of DNA at the DEJ, and such patients have a higher index of disease activity. ${ }^{1,14,15}$

\section{Conclusion}

The LBT is a useful diagnostic tool for LE patients. However, the correct interpretation of this test requires detailed knowledge of several correlates, such as the site of the biopsy (lesional or normal skin, sun-protected versus sun-exposed skin), deposit components, morphology and brightness of the immunofluorescent band, and other associated serologic findings, as well as the response to treatment. A positive LBT on sun-protected nonlesional skin represents a sensitive and specific criterion for identifying patients with LE. Furthermore, the LBT on sun-protected normal skin may be helpful in diagnosing SLE in patients with inconclusive clinical and serological profiles and may also be of prognostic significance, particularly if all three (IgG, IgM, and $\operatorname{IgA}$ ) immunoglobulins are found at the DEJ. Importantly, the LBT is a laboratory procedure that should always be interpreted in conjunction with clinical findings and other serological and immunopathological parameters in order to make a correct diagnosis.

\section{Disclosure}

The authors report no conflicts of interest in this work.

\section{References}

1. Crowson AN, Magro C. The cutaneous pathology of lupus erythematosus: a review. J Cutan Pathol. 2001;28:1-23.

2. McCarty DJ, Manzi S, Medsger TA Jr, Ramsey-Goldman R, LaPorte RE, Kwoh CK. Incidence of systemic lupus erythematosus. Race and gender differences. Arthritis Rheum. 1995;38:1260-1270.

3. Samanta A, Roy S, Feehally J, Symmons DP. The prevalence of diagnosed systemic lupus erythematosus in whites and Indian Asian immigrants in Leicester city, UK. Br J Rheumatol. 1992;31:679-682.

4. Mok CC, Mak A, Chu WP, To CH, Wong SN. Long-term survival of southern Chinese patients with systemic lupus erythematosus: a prospective study of all age-groups. Medicine (Baltimore). 2005;84: 218-224. 
5. Funauchi M, Shimadzu H, Tamaki C, et al. Survival study by organ disorders in 306 Japanese patients with systemic lupus erythematosus: results from a single center. Rheumatol Int. 2007;27:243-249.

6. Crowson AN, Magro CM. Cutaneous histopathology of lupus erythematosus. Diagn Histopathol. 2009;15:157-185.

7. Burnham TK, Neblett TR, Fine G. The application of the fluorescent antibody technic to the investigation of lupus erythematosus and various dermatoses. J Invest Dermatol. 1963;41:451-456.

8. Cormane RH. "Bound" globulin in the skin of patients with chronic discoid lupus erythematosus and systemic lupus erythematosus. Lancet. 1964;1:534-535.

9. Reich A, Meurer M, Viehweg A, Muller DJ. Narrow-band UVB induced externalization of selected nuclear antigens in keratinocytes: implications for lupus erythematosus pathogenesis. Photochem Photobiol. 2009;85:1-7.

10. Cardinali C, Caproni M, Fabbri P. The utility of the lupus band test on sun-protected non-lesional skin for the diagnosis of systemic lupus erythematosus. Clin Exp Rheumatol. 1999;17:427-432.

11. Mehta V, Sarda A, Balachandran C. Lupus band test. Indian J Dermatol Venereol Leprol. 2010;76:298-300.

12. Leibold AM, Bennion S, David-Bajar K, Schleve MJ. Occurrence of positive immunofluorescence in the dermoepidermal junction of sun-exposed skin of normal adults. J Cutan Pathol. 1994;21: 200-206.

13. Fabré VC, Lear S, Reichlin M, Hodge SJ, Callen JP. Twenty percent of biopsy specimens from sun-exposed skin of normal young adults demonstrate positive immunofluorescence. Arch Dermatol. 1991;127: 1006-1011.

14. Liu T, Liu FR. Prognostic value of lupus band test in unexposed normal skin of patients with systemic lupus erythematosus. Chin Med J. 1989;102:602-604.
15. Provost TT, Andres G, Maddison PJ, Reichlin M. Lupus band test in untreated SLE patients: correlation of immunoglobulin deposition in the skin of the extensor forearm with clinical renal disease and serological abnormalities. J Invest Dermatol. 1980;74:407-412.

16. Cardinali C, Caproni M, Fabbri P. The composition of the lupus band test (LBT) on the sun-protected non-lesional (SPNL) skin in patients with cutaneous lupus erythematosus (CLE). Lupus. 1999;8: 755-770.

17. Magro CM, Crowson AN, Harrist TJ. The use of antibody to C5b-9 in the subclassification of lupus erythematosus. Br J Dermatol. 1996;134: 855-862.

18. Magro CM, Crowson AN. The immunofluorescent profile of dermatomyositis. A comparative study with lupus erythematosus. J Cutan Pathol. 1997;24:543-552.

19. Magro CM, Dyrsen ME. The use of $\mathrm{C} 3 \mathrm{~d}$ and $\mathrm{C} 4 \mathrm{~d}$ immunohistochemistry on formalin-fixed tissue as a diagnostic adjunct in the assessment of inflammatory skin disease. J Am Acad Dermatol. 2008;59: 822-833.

20. Carvalho MF, Coelho RA. Lupus band test: diagnostic value in disseminated lupus erythematosus. Acta Med Port. 1991;4:242-248.

21. Shibeshi D, Blaszczyk M, Jarzabek-Chorzelska M, Jablonska S, Chorzelski T. Immunopathologic findings in systemic sclerosis patients: clinical and immunopathologic relationships. Int J Dermatol. 1989;28:650-656.

22. Davis BM, Gilliam AN. Prognostic significance of subepidermal immune deposits in uninvolved skin of patients with systemic lupus erythematosus: 10 year longitudinal study. J Invest Dermatol. 1984;83: 242-247.

23. Zecević RD, Pavlović MD, Stefanović D. Lupus band test and disease activity in systemic lupus erythematosus: does it still matter? Clin Exp Dermatol. 2006;31:358-360.
Therapeutics and Clinical Risk Management

\section{Publish your work in this journal}

Therapeutics and Clinical Risk Management is an international, peerreviewed journal of clinical therapeutics and risk management, focusing on concise rapid reporting of clinical studies in all therapeutic areas outcomes, safety, and programs for the effective, safe, and sustained use of medicines. This journal is indexed on PubMed Central, CAS,

\section{Dovepress}

EMBase, Scopus and the Elsevier Bibliographic databases. The manuscript management system is completely online and includes a very quick and fair peer-review system, which is all easy to use. Visit http://www.dovepress.com/testimonials.php to read real quotes from published authors. 